\title{
What have we learned from 8 years of deep brain stimulation of the anterior thalamic nucleus? Experiences and insights of a single center
}

\author{
*Elisabeth Kaufmann, MD, ${ }^{1}$ Kai Bötzel, MD, ${ }^{2}$ Christian Vollmar, MD, PhD, ${ }^{1}$ \\ Jan-Hinnerk Mehrkens, MD, ${ }^{3}$ and Soheyl Noachtar, MD1 \\ ${ }^{1}$ Epilepsy Center, Department of Neurology; and Departments of ${ }^{2}$ Neurology and ${ }^{3}$ Neurosurgery, University Hospital, LMU \\ Munich, Germany
}

\begin{abstract}
OBJECTIVE In the absence of a standard or guideline for the treatment of epilepsy patients with deep brain stimulation (DBS) of the anterior nucleus of the thalamus (ANT), systematic single-center investigations are essential to establish effective approaches. Here, the authors report on the long-term results of one of the largest single-center ANT DBS cohorts.
\end{abstract}

METHODS The outcome data of 23 consecutive patients with transventricularly implanted electrodes were retrospectively analyzed with regard to adverse events, lead placement, stimulation-related side effects, and changes in seizure frequency. Depression and quality-of-life scores were collected in a subgroup of 9 patients.

RESULTS All but 2 patients initially underwent bilateral implantation, and $84.4 \%$ of all DBS leads were successfully located within the ANT. The mean follow-up time was $46.57 \pm 23.20$ months. A seizure reduction $>50 \%$ was documented in $73.9 \%$ of patients, and $34.6 \%$ achieved an Engel class I outcome. In 3 patients, clinical response was achieved by switching the electrode contact or changing from the monopolar to bipolar stimulation mode. Unilateral implantation seemed ineffective, whereas bilateral stimulation with successful ANT implantation only on one side led to a clinical response. Double stimulation with additional vagus nerve stimulation was safe. Changes in cycling mode or stimulation amplitude influenced therapy tolerability and, only to a lesser extent, seizure frequency. Side effects were rare and typically vanished by lowering the stimulation amplitude or changing the active electrode contact. Furthermore, depression and aspects of quality of life significantly improved with ANT DBS treatment.

CONCLUSIONS The transventricular approach as well as double stimulation proved safe. The anteroventral ANT appeared to be the most efficacious stimulation site. This systematic investigation with reluctant medication changes allowed for the development of a better idea of the association between parameter changes and outcome in ANT DBS patients, but larger samples are still needed to assess the potential of bipolar stimulation and distinct cycling frequencies. Furthermore, more multifaceted and objective assessments of treatment outcome are needed to fully assess the effects of ANT DBS treatment. https://thejns.org/doi/abs/10.3171/2020.6.JNS20695

KEYWORDS deep brain; thalamus; epilepsy; neuromodulation; stimulation; functional neurosurgery

$\mathrm{D}$ EEP brain stimulation (DBS) targeting the anterior nucleus of the thalamus (ANT) was approved in 2010 in Europe and in 2018 in the United States for the treatment of adult patients with drug-resistant focal-onset epilepsy syndromes. It was established as a safe and well-tolerated third-line therapy option for patients in whom surgery or vagus nerve stimulation (VNS) fails or those who are not candidates for surgery due to un- known or multiple seizure origins. ${ }^{1-3}$ Its antiepileptic effect is based on the inhibition of seizure generalization, i.e., seizure propagation through the thalamus, as well as neuromodulatory mechanisms reducing the seizure frequency over time..$^{2-4}$ ANT DBS has the potential to significantly reduce the frequency and intensity of focal to bilateral tonic-clonic seizures and to significantly increase patients' quality of life (QOL)., ${ }^{2,3}$ It was reported that after

ABBREVIATIONS AED = antiepileptic drug; ANT = anterior nucleus of the thalamus; BDI = Beck Depression Inventory; DBS = deep brain stimulation; EEG = electroencephalography; IPG = implantable pulse generator; $\mathrm{QOL}=$ quality of life; QOLIE-31 = Quality of Life in Epilepsy 31; SANTE = Stimulation of the Anterior Nucleus of the Thalamus for Epilepsy; VAT = volume of activated tissue; VNS = vagus nerve stimulation.

SUBMITTED March 6, 2020. ACCEPTED June 11, 2020.

INCLUDE WHEN CITING Published online October 30, 2020; DOI: 10.3171/2020.6.JNS20695.

* J.H.M. and S.N. contributed equally to this work and share senior authorship. 
5 years, a seizure frequency reduction of more than $50 \%$ was achieved in $68 \%$ of patients, and $19 \%$ became seizure free. ${ }^{3}$ However, clinicians are challenged by the multitude of possible parameter settings and the numerous pending decisions regarding patient management. Until now, there has been no standard or guideline for ANT DBS treatment, and it is thus mainly guided by empirical approaches. The only prospective randomized controlled trial is the Stimulation of the Anterior Nucleus of the Thalamus for Epilepsy (SANTE) study with its 3-, 5-, and 7-year followup reports on the outcome of 110 ANT DBS patients..$^{2,3,6}$

At present, different approaches are used within and between studies regarding patient selection, imaging, ${ }^{7-10}$ surgical procedure, and parameter setting. Most centers use a transventricular approach, which proved to be similarly safe as the frontal extraventricular trajectory and was associated with a higher success rate in placing the lead within the small ANT. ${ }^{11,12}$ Recently, promising data were reported by American centers using an alternative parietal extraventricular approach, achieving a lead implantation success rate of $90 \% .{ }^{7,13}$ Uncertainty exists regarding the optimal stimulation parameters, which are thought to play a major role in determining the functional action of DBS. ${ }^{14}$ According to current knowledge, the effect of high-frequency stimulation $(>50 \mathrm{~Hz})$ is complex and multifaceted, with activating effects on neurons within $2 \mathrm{~mm}$ of the electrode and suppressing effects on neurons that are at least $2 \mathrm{~mm}$ away from the electrode. ${ }^{15}$ The activation generates axonal output at the stimulation frequency and seems to overwrite the intrinsic activity of the neuronal circuitry and to block pathological activity. ${ }^{16-18}$ Conflicting data have been reported regarding the effect of low-frequency stimulation, which can lead to a seizure frequency reduction on the one hand, ${ }^{19}$ but also induce seizures on the other hand. ${ }^{20,21}$ Current knowledge is mainly derived from DBS in patients with movement disorders and kindling rat models. Experience on distinct frequency settings in epilepsy patients with ANT DBS, however, is insufficient. Furthermore, stimulation is typically started in monopolar mode, but bipolar stimulation has the advantage of a more localized tissue activation and a lower risk of side effects. ${ }^{14,22} \mathrm{Few}$ studies also used modifications of the pulse width ${ }^{23-26}$ and/or the stimulation cycle, ${ }^{5,25-28}$ increasing the complexity of available ANT DBS data and reducing the comparability of study results.

Nevertheless, in the absence of a defined standard, systematic data acquisitions of single centers are more essential to establish effective protocols. Compared with earlier single-center studies, ${ }^{5,12,24-27,29-34}$ we report one of the largest single-center cohorts treated by the same core team with a standardized approach and with minimal antiepileptic drug (AED) changes, allowing for a more meaningful evaluation of DBS effects.

\section{Methods \\ Study Cohort}

All adult epilepsy patients who underwent ANT DBS implantation between August 2011 and June 2019 were included in the study. They were prospectively followed regarding their seizure frequency, seizure semiology, and stimulation side effects. Relevant clinical and demographic data were extracted from the patient files. The respective epilepsy syndromes were classified in a multidisciplinary case conference based on results of the presurgical multimodal diagnostic testing, including electroencephalography (EEG), seizure semiology, MRI, nuclear imaging, and neuropsychological testing. All patients were urged to use a seizure diary to objectify the therapy outcome.

Conditions for ANT DBS implantation were adult age, drug-resistant epilepsy, a complete video-EEG evaluation at our epilepsy monitoring unit, an unpromising surgical therapy option or unsuccessful resective surgery, and disabling seizures at least once a month (i.e., motor seizures with impaired awareness such as focal to bilateral tonicclonic seizures, hyperkinetic seizures, or astatic seizures). Contraindications encompassed severe depression; suicide attempts; history of psychosis, dementia, extensive parenchymal damage including the thalamus, or general surgery or anesthesia contraindications; and involvement in contact sports with a high risk of device damage.

\section{Surgical Procedures and Electrode Localization}

Implantation of the ANT DBS device was conducted in a two-step procedure. First, the stimulation electrodes (model 3387, 1.27-mm lead body diameter, contact length $1.5 \mathrm{~mm}$, intercontact distance $1.5 \mathrm{~mm}$; Medtronic) were stereotactically implanted into the ANT. A Zeppelin stereotactic head frame (adeor medical AG) was applied to the patient's skull, and a full head CT scan was obtained with a 1-mm slice thickness.

The implantation planning was based on the coregistered stereotactic CT and preoperative 3T high-resolution MRI head scans. The ANT was always approached transventricularly, setting the preliminary target at 12 $\mathrm{mm}$ superior, 5-6 $\mathrm{mm}$ lateral, and $0-2 \mathrm{~mm}$ anterior to the midcommissural point. These standard target coordinates were adjusted based on individual anatomical landmarks, aiming at an anterior and superior electrode position within the ANT. A postoperative high-resolution CT scan was acquired to rule out intracranial bleeding and to confirm the electrode position. In a second surgery, typically performed 2 days later, the thalamic stimulation electrodes were extended and subcutaneously connected with a nonrechargeable, fully implantable pulse generator (IPG; Activa PC; Medtronic) located in a subcutaneous infraclavicular or abdominal pouch. Both surgeries were performed under general anesthesia and without microelectrode recording. The antiepileptic medication remained unchanged, except in patients with coagulopathy who were on a regimen of valproate.

The anatomical location of the stimulation electrodes was verified via coregistration of the preoperative MRI and postoperative CT or MRI scans. The coregistration and 3D visualization was performed using Amira (Thermo Fisher Scientific) and a MATLAB-based open-source toolbox (Lead DBS). ${ }^{35}$ The latter approach included a transformation into the Montreal Neurological Institute standard stereotactic space using the SyN registration method as implemented in Advanced Normalization Tools. ${ }^{36}$ The DISTAL atlas $^{37}$ was used for 3D visualization of the lead trajectories and their relative position to thalamic subnuclei. 


\section{Stimulation Initiation and Therapeutic Strategies}

The stimulation device was usually activated 3 weeks after implantation. First, the electrode impedance was tested to screen for hardware failures such as a cable break or lead disconnection. Afterward, the stimulation device was activated using a cyclic mode with 1 minute $\mathrm{ON}$ and 5 minutes OFF, a pulse width of $90 \mu \mathrm{sec}$, and a stimulation frequency of 140-145 Hz. A monopolar stimulation mode was used, except for patients with cardiac or gastric pacemakers or ongoing VNS. The electrode contacts with the most superior and anterior localization within the ANT were used as active contacts. The stimulation amplitude was set between 1.5 and $3.5 \mathrm{~V}$, depending on the resulting therapeutic impedance and side effects. The desired initial therapeutic impedance was between 3 and $4 \mathrm{~mA}$, with similar values on both sides. In case of an immediate occurrence of putative stimulation-related side effects such as paresthesia, vertigo, concentration difficulties, trouble finding words, increase in seizure frequency, or prolonged aura sensation, the stimulation amplitude was lowered by $0.5-1 \mathrm{~V}$ or until the side effect vanished. If an implantation effect occurred postsurgically, the initial stimulation amplitude was typically set lower (1.5-2.5 V). If the stimulation was tolerated well, the stimulation amplitude was increased to $1.5-5 \mathrm{~V}$ the next day (i.e., a therapeutic impedance of 4-6 mA). During the follow-up visits 3, 9, and 15 months later and then yearly, the stimulation amplitude was modified if clinically required. The anticonvulsive medication remained unchanged unless clinically unavoidable.

\section{Seizure Semiology and Frequency}

The seizure semiology was classified based on the video recordings acquired during the video-EEG monitoring as well as the patients' and their relatives' descriptions. The seizure frequency was extracted from the patients' seizure records and an additional interview.

\section{Questionnaire-Based Outcome Measurement}

Established questionnaires were used to assess aspects of depression and QOL in a subgroup of 9 patients at baseline and during the follow-up visits. The questionnaire battery encompassed the Beck Depression Inventory (BDI), the SF-36, and Quality of Life in Epilepsy 31 (QOLIE-31).

\section{Statistical Analysis}

The mean and standard deviation or the $95 \%$ confidence interval of the mean were calculated for quantitative parameters. A Kolmogorov-Smirnov test was performed to test for normal distribution, and an F-test was used to compare group variances. Group differences were calculated using t-tests or the Mann-Whitney U-test or Wilcoxon signed-rank test in case normal distribution was not given; $\mathrm{p}<0.05$ was considered significant.

\section{Results}

\section{Clinical Characteristics}

A total of 23 patients with drug-resistant epilepsy underwent DBS implantation between August 2011 and June
2019 with 1 to 6 implantations per year. Patient sex was nearly equally represented, with 10 female and 13 male patients. The mean age at implantation was $35.48 \pm 11.62$ years (range 18-51 years). At the time of DBS surgery, the patients had a mean disease duration of $23.74 \pm 14.93$ years (minimum 3 years; maximum 50 years) and were treated with a mean of $2.83 \pm 1.03$ AEDs.

All patients underwent presurgical evaluation in our video-EEG monitoring unit, including intracranial EEG recording with stereo-EEG or electrocorticography in $34.8 \%(8 / 23$ patients) and nuclear imaging in 56.5\% (13/23 patients). Of note, 5 patients $(21.7 \%)$ had prior surgery and 8 patients $(34.8 \%)$ had prior VNS with insufficient clinical response. Similar to the SANTE approach, the VNS device was explanted in the first 3 patients on DBS implantation. The helical lead around the vagus nerve was left in situ to reduce the risk of an iatrogenic nerve lesion on adhesion removal but leading to MRI incompatibility. In the remaining 5 patients, the VNS devices were left in situ to maintain MRI compatibility and the option of later reactivation. In 3 of these patients, the IPG battery was empty and was not replaced, because no clinical effect was observed under prior VNS therapy. Two of these 3 patients experienced a significant reduction in seizure frequency with ANT DBS treatment, but the third patient did not respond to treatment and thus VNS reactivation in addition to the ANT DBS stimulation is planned as a prospective treatment option. The other 2 patients received ANT DBS therapy in addition to ongoing VNS therapy because they attributed some clinical effect to their VNS therapy, with one of these patients responding to treatment after double stimulation.

The majority of patients were diagnosed with a bilateral epilepsy syndrome $(n=13 ; 56.5 \%)$. In one case, the epilepsy syndrome was classified as generalized epilepsy due to a Lennox-Gastaut syndrome and additional cerebral postradiation changes. The remaining 9 patients (39.1\%) had a lateralized epilepsy syndrome, whereby 5 had a right and 4 had a left hemispheric seizure onset. In detail, 3 patients had a temporal (in 2 of 3 a bitemporal) focus, 6 had a frontal (in 4 of 6 a bifrontal) focus, and 1 had a temporooccipital seizure focus. Another 4 patients had a lateralized but not localized seizure focus, and 8 patients were multifocal (7 of 8 bilateral). Nine patients (39.1\%) had a history of encephalitis or meningitis, and 7 patients (30.4\%) had a hereditary epilepsy etiology (focal cortical dysplasia [n = $6]$ and a bioccipital double cortex $[\mathrm{n}=1])$. Hippocampal sclerosis was described in 3 of them. A summary of the clinical and demographic data is given in Table 1.

Most of the patients $(17 / 23 ; 73.91 \%)$ had focal to bilateral tonic-clonic seizures as their most severe seizure type. A preceding aura occurred in 9 patients (39.1\%), and $2(8.7 \%)$ had a history of additional psychogenic seizures.

Five patients were intellectually disabled due to a microcephaly, autism, extensive perinatal ischemia, or encephalitis at a young age. Furthermore, 3 patients were prediagnosed with mild or moderate depression.

\section{DBS Implantation and Electrode Localization}

In all but 2 patients, the ANT was implanted bilaterally. In 1 patient, implantation was performed only contralateral 
TABLE 1. Cohort characteristics stratified by the amount of seizure reduction

\begin{tabular}{|c|c|c|c|c|c|c|c|c|c|c|}
\hline $\begin{array}{c}\text { Case } \\
\text { No. }\end{array}$ & $\begin{array}{c}\text { Age at } \\
\text { Implantation } \\
\text { (yrs), Sex }\end{array}$ & $\begin{array}{c}\text { Age at } \\
\text { Disease } \\
\text { Onset (yrs) }\end{array}$ & Syndrome & $\begin{array}{c}\text { Hemisphere } \\
\text { Affected }\end{array}$ & Etiology & VNS & $\begin{array}{c}\text { VNS } \\
\text { Explanted }\end{array}$ & $\begin{array}{c}\text { Prior } \\
\text { Surgery }\end{array}$ & $\begin{array}{c}\text { No. of } \\
\text { AEDs at } \\
\text { Implantation }\end{array}$ & $\begin{array}{c}\text { Seizure } \\
\text { Reduction }\end{array}$ \\
\hline 9 & $50, \mathrm{~F}$ & 3 & Focal & Rt & $\mathrm{HS} \mathrm{rt}>\mathrm{It}$ & No & & Yes & 1 & \multirow{5}{*}{$>90 \%$} \\
\hline 20 & $37, \mathrm{M}$ & 24 & Multifocal & Bilateral & Encephalitis & No & & No & 2 & \\
\hline 1 & $45, M$ & 11 & Multifocal & Bilateral & Gliosis & Yes & Yes & No & 3 & \\
\hline 3 & $48, M$ & 16 & Focal & $\mathrm{Lt}$ & Traumatic brain injury & No & & No & 3 & \\
\hline 5 & $32, F$ & 7 & Focal & $\mathrm{Rt}$ & Double cortex rt $>$ It & No & & No & 2 & \\
\hline 2 & $32, F$ & 8 & Generalized & & Lennox-Gastaut, radiation & Yes & Yes & No & 2 & \multirow{12}{*}{$>50 \%$} \\
\hline 4 & $39, \mathrm{M}$ & 17 & TLE & Bilateral & Suspected meningitis & Yes & Yes & No & 4 & \\
\hline 23 & $26, M$ & 22 & Multifocal & Bilateral & Encephalitis & No & & No & 1 & \\
\hline 6 & $29, M$ & 11 & TLE & $\mathrm{Lt}$ & FCD IIIa & No & & Yes & 2 & \\
\hline 7 & $18, M$ & 9 & FLE & Bilateral & FCD & No & & No & 3 & \\
\hline 8 & $51, \mathrm{M}$ & 11 & TOLE & $\mathrm{Rt}$ & Suspected encephalitis & Yes & No & Yes & 2 & \\
\hline 21 & $26, M$ & 12 & Multifocal & Bilateral & FCD, microcephaly & Yes & No & No & 4 & \\
\hline 10 & $44, \mathrm{M}$ & 34 & FLE & Bilateral & Suspected encephalitis & No & & No & 1 & \\
\hline 11 & $19, F$ & 2 & Multifocal & Bilateral & Encephalitis & No & & No & 4 & \\
\hline 19 & $51, \mathrm{M}$ & 1 & Multifocal & Bilateral & Encephalitis, rt HS & No & & No & 4 & \\
\hline 17 & $25, F$ & 21 & Focal & $\mathrm{Rt}$ & Perinatal ischemia & No & & No & 3 & \\
\hline 15 & $18, F$ & 15 & Multifocal & Bilateral & Unknown & No & & No & 3 & \\
\hline 13 & $46, F$ & 1 & FLE & $\mathrm{Lt}$ & Lt precentral gliosis, encephalitis & Yes & No & No & 3 & \multirow{6}{*}{$<50 \%$} \\
\hline 18 & $28, F$ & 8 & FLE & Bilateral & FCD & No & & Yes & 4 & \\
\hline 14 & $40, M$ & 4 & Multifocal & Rt & Encephalitis & Yes & No & No & 4 & \\
\hline 16 & $19, F$ & 12 & FLE & Bilateral & FCD & No & & No & 4 & \\
\hline 12 & $48, M$ & 4 & FLE & $\mathrm{Lt}$ & FCD & No & & Yes & 3 & \\
\hline 22 & $45, \mathrm{~F}$ & 17 & TLE & Bilateral & $\mathrm{Rt}+\mathrm{It} \mathrm{HS}$ & Yes & No & No & 3 & \\
\hline
\end{tabular}

FCD = focal cortical dysplasia; FLE = frontal lobe epilepsy; HS = hippocampal sclerosis; TLE = temporal lobe epilepsy; TOLE = temporooccipital lobe epilepsy.

to the seizure focus due to extensive parenchymal damage after a perinatal infarct. In another patient with bilateral epilepsy, electrode implantation was at first implemented only on one side because of a prominent thalamic vein and thus a high bleeding risk. However, since this patient did not respond to the treatment, a second electrode was implanted 1.5 years later without any complications. The IPG was placed in the right pectoral region in 22 patients (95.7\%). One young female patient preferred an abdominal IPG implantation.

In 17 of 23 patients (73.9\%), the DBS electrodes were bilaterally located within the ANT, and in 4 patients $(17.4 \%)$ the ANT was hit on only one side, including the patient with unilateral DBS electrode implantation. All 4 patients had a unilateral seizure focus, and successful ANT DBS electrode implantation was contralateral in 3 of them. In 2 patients, both ANTs were missed. In summary, 7 of the implanted 45 DBS electrodes (15.6\%) were not within the target.

\section{Stimulation Settings and Modifications}

In the first 2 patients who underwent implantation, the ANT DBS device was activated the day after implantation. In the subsequent patients, DBS was started 3 weeks after surgery at the earliest. The activation was further postponed in 2 patients, because of an extensive implanta- tion effect or an unplanned gastrointestinal surgery. The follow-up time since implantation was on average $46.57 \pm$ 23.20 months (range 3-95 months).

DBS stimulation was always started with a pulse width of $90 \mu \mathrm{sec}$ and monopolar setting. A bipolar stimulation setting was only used in 3 patients with double stimulation (i.e., ANT DBS plus VNS or a gastric pacemaker) to create a more focused field of stimulation and to avoid interference with the other stimulation device. However, in 8 patients the stimulation setting was changed from monoto bipolar during the follow-up visits, because of insufficient or decreasing clinical response or side effects. At the last follow-up, the mean stimulation amplitude was 4.07 $\mathrm{V}$ (minimum $2.4 \mathrm{~V}$; maximum $5.4 \mathrm{~V}$ ) for monopolar and $4.70 \mathrm{~V}$ (minimum $3.0 \mathrm{~V}$; maximum $7.0 \mathrm{~V}$; $\mathrm{p}=0.070$ ) for bipolar stimulations. Therapeutic impedances were available for 20 patients, averaging $690.00 \pm 249.35 \Omega(5.89$ $\pm 1.44 \mathrm{~mA}$ ) in patients with monopolar stimulation and $790.78 \pm 294.58 \Omega(6.19 \pm 1.37 \mathrm{~mA} ; \mathrm{p}=0.520)$ in patients with bipolar stimulation. A second stimulation group with lower stimulation amplitude was programmed in 2 patients for nocturnal use to mitigate stimulation-related arousals during night sleep.

The cycling mode with an ON phase of 1 minute and an OFF phase of 5 minutes was always switched on at the time of stimulation initiation. The cycling frequency was 


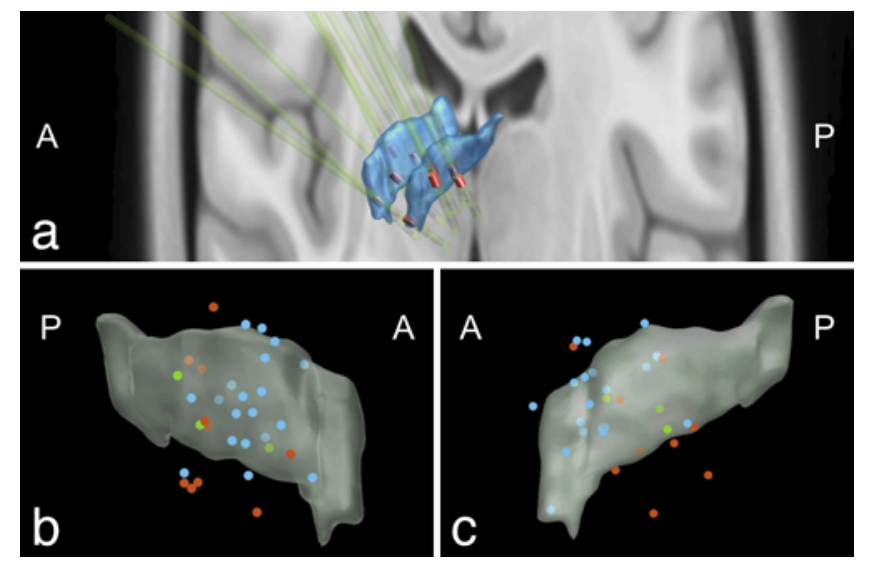

FIG. 1. Position of active electrode contacts in relation to the ANT. a: A reconstruction of the leads placed in the 5 patients with more than $90 \%$ reduction in seizure frequency. The active electrode contacts are highlighted in red. The ANT is visualized in blue. $\mathbf{b}$ and $\mathbf{c}$ : Lateral views of the right ANT (b) and left ANT (c). The positions of the active electrode contacts of the entire group are shown, whereby nonresponders are highlighted in red, responders in blue, and the 3 patients with Engel class I outcome in green. $A=$ anterior; $P=$ posterior. Figure is available in color online only.

later changed in 2 patients with no or insufficient clinical response: a cycling frequency of 1 minute $\mathrm{ON}$ and 3 minutes OFF did not lead to a clinical improvement and both patients complained about restlessness. Continuous stimulation was temporarily used in one of these patients, leading to a clear clinical improvement but quick IPG depletion. In addition to this patient, only one other patient required IPG replacement after 5 years of stimulation.

\section{Long-Term Follow-Up Efficacy}

In half of the patients $(12 / 23 ; 52.2 \%)$ the antiepileptic medication remained unchanged over the entire follow-up period. In 4 patients, the medication was extended by one additional AED, and in the other 7 patients, one of the initial AEDs was replaced by another AED.

The majority of patients $(17 / 23 ; 73.9 \%)$ were classified as responders, as a frequency reduction $>50 \%$ of their most severe seizure type was observed with ANT DBS treatment; 9 of these patients had no changes to their antiepileptic medication regimen. The position of their active contacts is shown in Fig. 1. Favorable clinical outcome was typically preceded by an insertional effect and expanded over time. Three of the patients were nonresponders in the beginning and became responders after the stimulation contacts were changed or they switched to bipolar stimulation. The most severe seizure type was focal to bilateral tonic-clonic in 14 of the 17 responders and bilateral tonic or hyperkinetic in the other 3 patients. The 5 patients who did not respond to treatment experienced focal to bilateral tonic-clonic seizures $(\mathrm{n}=3)$, focal onset seizures with automatisms $(n=1)$, or hyperkinetic $(n=1)$ or hypnopompic $(n=1)$ seizures as their most severe seizure type.

An Engel class I outcome was achieved in $13.6 \%$ (3/22) of the patients with a follow-up period of more than 1 year; of these, 1 patient had a bilateral multifocal epilepsy syndrome due to encephalitis; the other 2 patients were classified as having right hemispheric epilepsy syndromes with occipital double cortex or bilateral hippocampal sclerosis. In 2 of these patients, the DBS electrodes were bilaterally within the ANT, and in the third patient, the stimulation electrodes hit the ANT on only one side (Fig. 1). No association was observed between clinical response and disease duration or age at implantation.

Longitudinal questionnaire-based data on depression and QOL were available in 9 patients, with half of them $(5 / 9 ; 55.6 \%)$ being responders and the other half (4/9; $44.4 \%$ ) being nonresponders. Two and a half years after ANT DBS implantation, a significant reduction in the depression score (BDI) was observed (baseline 95\% CI 4.84-17.38 and follow-up 95\% CI 1.68-13.88; $\mathrm{p}<0.0001$ ) (Fig. 2). Furthermore, the parameter "general health" on the SF-36 questionnaire significantly improved by 18.3 points compared with baseline ( $\mathrm{p}<0.0001$ ) (Fig. 2). None of the other SF-36 subcategories or any of the QOLIE-31 questionnaire revealed a significant modification with the patient receiving ANT DBS treatment.

\section{Side Effects}

One patient could not undergo implantation via a transventricular or an extraventricular approach (the patient was sent to another center experienced with the extraventricular approach) due to prominent vessels and thus a high intraoperative bleeding risk. The implantations in the 23 patients were performed without pre- or intraoperative complications, but one of the patients presented with a scapula alata at the first follow-up visit 3 weeks postimplantation. Most likely, the nervus thoracicus longus was affected during the subcutaneous tunneling of the lead, but no functional deficit remained after intense physiotherapy.

Stimulation activation and modification was typically tolerated well. Mild side effects were reported in individual patients: 1 patient presented with dizziness and problems finding words after a quick increase of the stimulation amplitude to $5 \mathrm{~V}$. Vertigo with swaying stand was observed in another patient with monopolar stimulation at $5 \mathrm{~V}$. In both patients, the side effects disappeared when the stimulation amplitude was lowered. Cerebral tingling paresthesia was associated with a rapid voltage increase in another and was avoided by a slow stepwise voltage elevation of $0.3-0.5 \mathrm{~V}$ per visit. A persistent aura $(\mathrm{n}=2)$ or an increase in the frequency of habitual seizures $(n=1)$ on stimulation activation or amplitude increase was observed, especially in patients with good implantation effect. Lowering the stimulation amplitude always resolved these epileptic side effects. The stimulation amplitudes were slowly increased again during the follow-up visits, if clinically required. This was then typically tolerated well. In 2 of the patients, a good clinical response (ultimate seizure frequency reduction by $84 \%$ and $88 \%$ ) was already achieved with low stimulation amplitudes of $2.9 \mathrm{~V}$ and $3.5 \mathrm{~V}$. However, the third patient remained a nonresponder (30\% seizure frequency reduction), although he tolerated higher stimulation amplitudes (4.2 V) during the course of the follow-up. As mentioned earlier, stimulation-induced arousals were mitigated by reducing the stimulation amplitude during the night $(\mathrm{n}=2)$.

Depression was documented in 2 patients who were 
Beck Depression Inventory

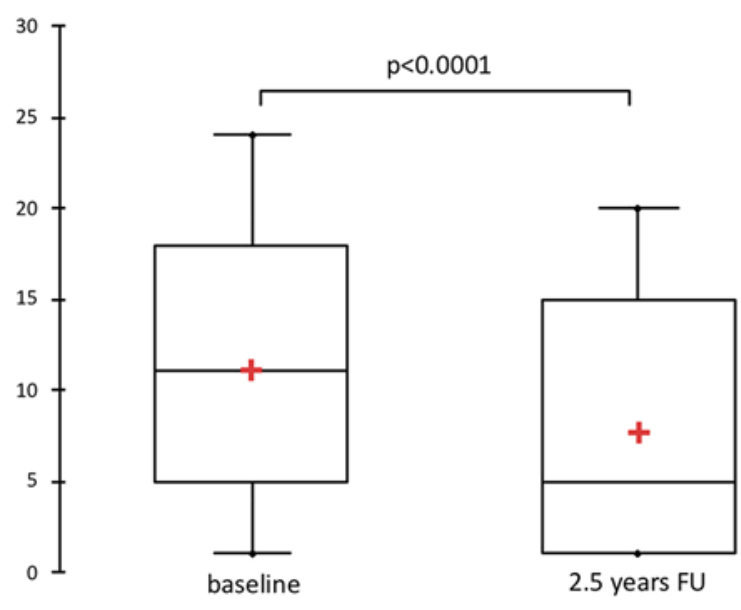

SF36 'general health'

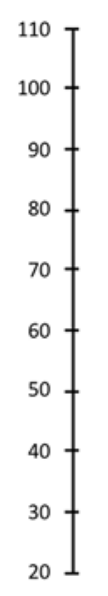

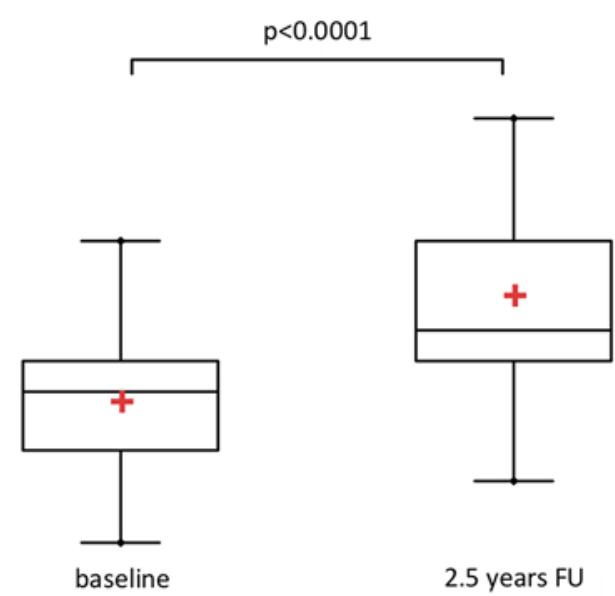

FIG. 2. Questionnaire-based outcome under ANT DBS treatment. The box plot figures visualize the significant improvement of the $\mathrm{BDI}$ scores (left) and the SF-36 subcategory general health (right) after 2.5 years of ANT DBS treatment compared with baseline $(n=9)$. The plus sign represents the mean, the box depicts the median and the first and third quartiles, and the bars represent the minimum and maximum values. $\mathrm{FU}=$ follow-up. Figure is available in color online only.

prediagnosed with depression; it was attributed to VNS explantation $(\mathrm{n}=2)$ and private stress factors $(\mathrm{n}=1)$. Their stimulation amplitudes were moderate $(3.0 \mathrm{~V}$ and $3.2 \mathrm{~V}$ ), and their active contacts were bilaterally located within the ANT $(n=1)$ or lateral and inferior to the ANT within the ventral lateral thalamus $(n=1)$. In the latter case, the active contacts were switched to the uppermost contacts, which were located adjacent to the ANT, and both patients received antidepressant drugs. One of these patients also reported memory impairment, which resolved after successful pharmacological treatment of his depression.

After 3 years and 7 years of good clinical response, 2 patients had to undergo explantation due to bacterial meningitis or postauricular dermal defects in the lead area. Neither patient has yet undergone reimplantation, because one patient recently underwent explantation and the other patient experienced a persistent seizure reduction outlasting DBS explantation. ${ }^{38}$

\section{Clinical Long-Term Experience and Approach Adjustments}

Over the course of time, we refined our implantation approach from a mainly coordinate-guided ANT implantation toward direct targeting aiming at a lead placement within the anterior portion of the ANT. This approach modification was motivated by a publication of Lehtimäki et al.,12 suggesting that stimulation within the anterior portion of the ANT might be associated with a more favorable outcome. Improved MRI protocols with thin-slice STIR sequences helped to visualize the ANT and ease the implantation planning. As our neurosurgeon has multiannual experience with DBS lead implantations, we did not observe an obvious improvement in the number of successful implantations or the responder rate. Being unexperienced with the stimulation configuration for ANT DBS in 2011, we set the stimulation parameters according to the SANTE parameters. The first patient cases then taught us to start with lower stimulation amplitudes and use bilevel stimulation in individual cases to avoid side effects and increase tolerability. Furthermore, measurements of the therapeutic impedance were integrated in our follow-up routine, because it better reflects the transmitted stimulation power compared with the stimulation amplitude. Of note, we did not observe adverse effects under double stimulation with VNS and DBS, which could thus be a valuable treatment option for patients who improved with VNS treatment. Our final patient management strategy is visualized in Fig. 3.

\section{Discussion}

We here report the outcome data of one of the largest single-center ANT DBS cohorts. ${ }^{39}$ Of note, all of the ANT DBS patients have been treated by a consistent interdisciplinary team that used the same (i.e., transventricular) implantation approach. Furthermore, in half of the cohort, the AEDs remained unchanged over the entire follow-up period, increasing the significance of the observations.

\section{Lead Placement and Long-Term Outcome}

The investigated cohort represents a typical sample of ANT DBS candidates, that is, adult patients with longlasting multidrug-resistant focal epilepsy syndromes with disabling seizures of bilateral or multifocal seizure origin. Despite the fear that the transventricular implantation approach might be associated with a relevant risk for intraventricular bleeding or secondary lead misplacement, no such complication was observed. However, $15.6 \%$ of the implanted stimulation electrodes were outside the ANT. Nevertheless, a responder rate of $74 \%$ was achieved with seizure freedom in $14 \%$, which is in line with the results of the SANTE trial $(69 \%$ of patients responded to treatment and 19\% experienced seizure freedom) $)^{2,3}$ and other smaller single-center reports. ${ }^{10,12,29,40,41}$ Precise electrode 


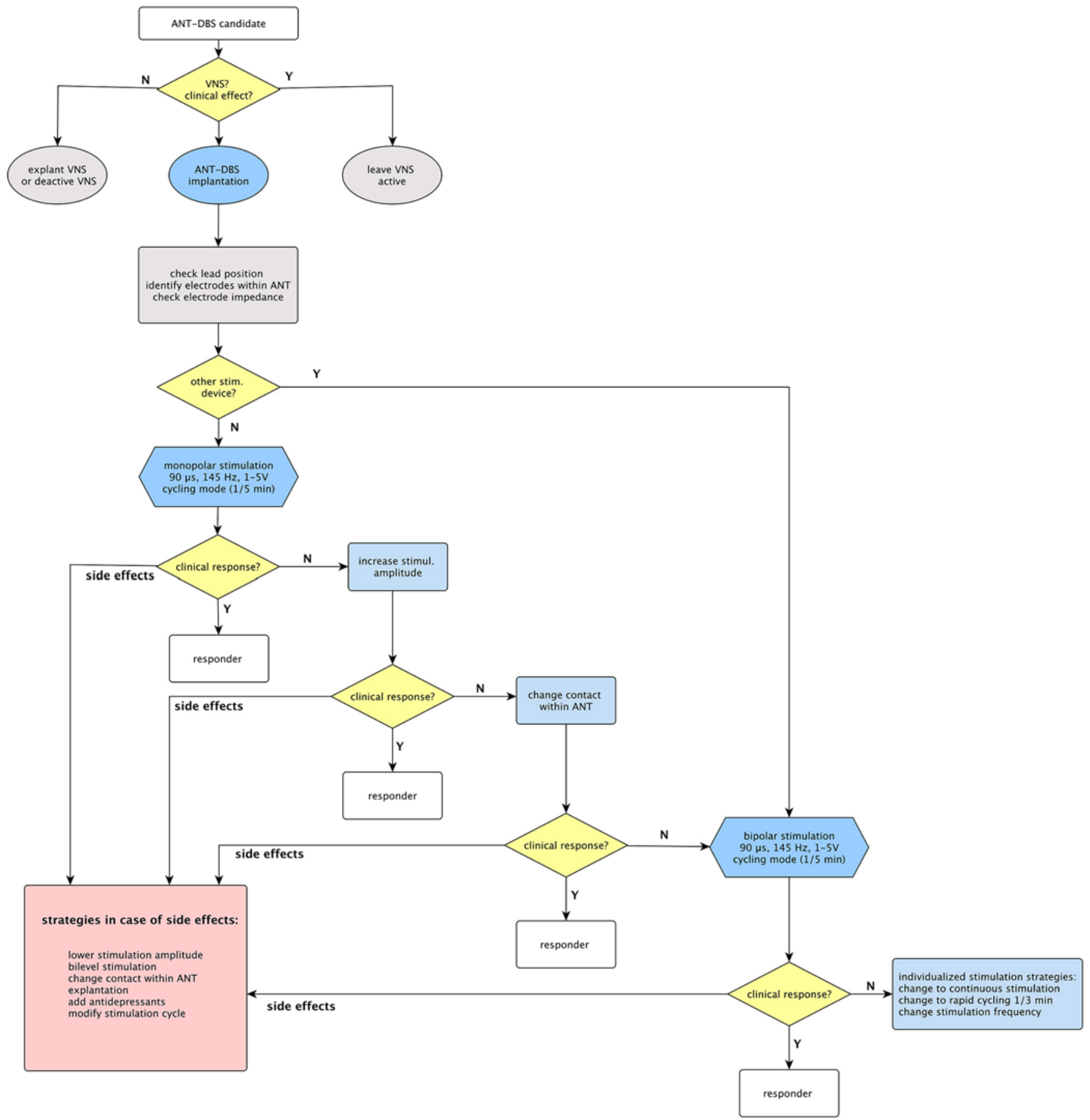

FIG. 3. Flowchart demonstrating the patient management strategy. $\mathrm{N}=$ no; stimul. = stimulation; $\mathrm{Y}=$ yes. Figure is available in color online only.

placement within the target is supposed to be crucial for a satisfactory clinical response. ${ }^{12}$ In accordance with a previous study on patients with transventricular ANT DBS, ${ }^{12}$ a favorable outcome was more likely if the volume of activated tissue (VAT) involved aspects of the ANT that were more superior and anterior. However, in our study, the rate of successful bilateral ANT implantations was similar in responders and nonresponders, and good clinical response was also achieved in patients in whom only one stimulation electrode was located within the ANT. This implicates that additional parameters may be essential or that thalamic structures neighboring the ANT are similarly effective. On the one hand, we were not able to reveal associations between outcome and clinical or demographic factors due to the small sample size of 23 patients. On the other hand, good outcome was observed in patients of dif- 
ferent age, disease duration, seizure origin, etiology, and semiology, underlining the effectiveness of ANT DBS in diverse focal-onset epilepsy syndromes. Furthermore, ANT DBS was comparably effective in patients with prior surgery, VNS, or none or both.

\section{Stimulation Amplitude}

In line with the experience from DBS in movement disorders and single investigations in epilepsy, ${ }^{25,26,29}$ stimulation amplitudes $<5 \mathrm{~V}$ were already sufficient to achieve a reduction in seizure frequency, especially in patients with a good insertional effect. We recommend deviating from the SANTE amplitude of $5 \mathrm{~V}$ and adjusting the voltage individually according to the impedance and occurrence of side effects, starting low and increasing the amplitude according to the clinical response. Lower stimulation amplitudes go along with a lower risk of side effects, increase the DBS therapy tolerability, and extend the IPG battery life. The battery life exceeded 5 years in our cohort. Higher stimulation amplitudes might be required in patients with electrode misplacement in order to achieve a larger VAT, as well as in patients with high impedance values.

\section{Monopolar Versus Bipolar Stimulation Mode}

Bipolar stimulation is thought to generate a more localized electrical field than monopolar stimulation and may thus have the potential to induce a more pronounced clinical effect with a lower risk of side effects. ${ }^{24}$ However, the responder rate was obviously higher in the monopolar group (12/13 vs $5 / 10$ patients), as we switched to bipolar mode mainly in cases of insufficient clinical response. The switch to bipolar stimulation did not lead to any side effects or significant changes in stimulation amplitudes or therapeutic impedance measures. However, 1 patient responded to treatment after being changed to bipolar stimulation. Although the full benefits of bipolar stimulation remain to be determined, it is a valuable option for patients with unsatisfactory outcomes or side effects under monopolar stimulation. More precise and directed stimulation might soon be available with the introduction of segmented electrodes for ANT DBS.

\section{Pulse Width, Frequency, and Stimulation Cycle}

More data and systematic investigations are needed to assess the impact of different pulse widths, frequencies, and stimulation application modes (i.e., continuous vs different intermittent stimulation modes). We did not modify the stimulation pulse width and frequency in our cohort to minimize the number of investigated parameters. Furthermore, animal studies have suggested that the anticonvulsant effects are dependent on the stimulation intensity rather than the frequency. ${ }^{21,42}$ So far, only one study has compared cycling and continuous stimulation in 4 patients with epilepsy and did not reveal any significant difference in regard to seizure frequency. ${ }^{24}$ In our cohort, increasing the cycling frequency seemed to cause restlessness, but continuous stimulation was well tolerated and might be more efficient than cyclic stimulation in patients with high seizure frequencies or seizure clusters. ${ }^{21}$

\section{Instructive Cases}

A systematic evaluation of every possible parameter setting is not feasible, as thousands of parameter combinations exist. Thus, evidence is mainly based on trial and error (i.e., instructive patient cases). In our cohort, nonresponders turned into responders by a change in the stimulation contacts and not by only increasing the stimulation amplitude or modifying the cycling mode. The stimulation contact is typically chosen based on the CT and MRI findings, and polysomnography was recently reported as an alternative biomarker-based approach to identify the most effective stimulation contact in patients with extraventricularly implanted DBS electrodes. ${ }^{30}$ Surprisingly, stimulation-related arousals were also observed in our patients with transventricular implantations, which challenges the hypothesis of an underlying activation of the reticular activating system. $^{30,43}$ Systematic polysomnographic investigations are needed to prove whether this is a systematic effect and associated with clinical outcome, but bilevel stimulation with lower stimulation amplitudes during the night should be considered in patients with transventricular implantation experiencing disturbed nights of sleep. A stimulationrelated increase in seizure frequency or the occurrence of a persistent aura, however, appeared to be a positive predictor for a favorable outcome. Furthermore, double stimulation with VNS and DBS was safe and effective and has the advantage of an antidepressant effect in addition to seizure frequency reduction. We are not aware of other studies on double stimulation (VNS plus ANT DBS); thus, its additive anticonvulsive potential has to be substantiated. In line with animal data, ${ }^{21,44}$ unilateral implantation and stimulation elicited no or a less pronounced anticonvulsant effect compared with bilateral stimulation. Therefore, the bilateral aspect appears to be essential, considering that 3 of 4 patients with only one-sided successful ANT implantation but bilateral thalamic stimulation became responders. As published previously, ANT DBS has the potential to disturb the seizure spread, to shorten the seizure duration, and to lower the seizure frequency. ${ }^{4}$ Furthermore, neuromodulatory effects seemed to endure DBS explantation for years. ${ }^{38}$

\section{Side Effects and Questionnaire-Based Outcome Measurement}

Only mild to moderate stimulation-related side effects occurred, and they always vanished when the stimulation amplitude was lowered. The large VAT likely affected neighboring anatomical structures and elicited side effects in ANT DBS patients with high stimulation amplitudes. Tolerability was increased with a stepwise elevation of the stimulation amplitude, putatively due to neuromodulatory processes and thus adaptions of the neuronal activation thresholds. In addition, a gradual impedance decrease ${ }^{45}$ might cause a successive increase in the effective current in patients with voltage-guided stimulation.

According to the subjective questionnaire-based outcome measurements, the patients' affect and general health significantly improved with ANT DBS treatment. However, it fell short of our interview-based impressions. The discrepancy might be explained by the observation that responders have a more critical and aware experience of their symptoms and limitations, impairing their ques- 
tionnaire results and limiting the comparability with baseline measurements. ${ }^{6}$

\section{Limitations}

Our single-center study is limited by the small size of the patient cohort and its inhomogeneity. Thus, subcohort analyses were mostly underpowered. Furthermore, the outcome of ANT DBS treatment was mainly assessed by evaluation of seizure diaries. However, documentation with seizure diaries is afflicted by inaccuracy, especially in patients with nocturnal seizures and postictal amnesia. Furthermore, the measurement of seizure frequency changes and the occurrence of side effects fall short of the multifaceted treatment outcome, such as changes in the activity level, sleep quality, attenuation of seizure severity, and associated constraints. Future studies should expand the number of evaluated outcome parameters and use, for example, integrative seizure detection devices to objectify and quantify alterations in epileptic activity.

\section{Conclusions}

We report one of the largest single-center studies on ANT DBS treatment, with up to 8 years of follow-up, and confirmed the safety and efficacy of the transventricular approach as well as double stimulation. The optimization of the VAT seemed to be essential for a favorable outcome, and tolerability was increased by an individual stepwise elevation of the stimulation amplitude. More multifaceted and objective assessments of treatment outcome as well as functional neuroanatomical correlations will hopefully provide a deeper understanding of the underlying pathophysiological mechanisms in the near future.

\section{References}

1. Gooneratne IK, Green AL, Dugan P, et al. Comparing neurostimulation technologies in refractory focal-onset epilepsy. J Neurol Neurosurg Psychiatry. 2016;87(11):1174-1182.

2. Fisher R, Salanova V, Witt T, et al. Electrical stimulation of the anterior nucleus of thalamus for treatment of refractory epilepsy. Epilepsia. 2010;51(5):899-908.

3. Salanova V, Witt T, Worth R, et al. Long-term efficacy and safety of thalamic stimulation for drug-resistant partial epilepsy. Neurology. 2015;84(10):1017-1025.

4. Hartl E, Feddersen B, Bötzel K, et al. Seizure control and active termination by anterior thalamic deep brain stimulation. Brain Stimul. 2017;10(1):168-170.

5. Piacentino M, Durisotti C, Garofalo PG, et al. Anterior thalamic nucleus deep brain Stimulation (DBS) for drugresistant complex partial seizures (CPS) with or without generalization: long-term evaluation and predictive outcome. Acta Neurochir (Wien). 2015;157(9):1525-1532.

6. Tröster AI, Meador KJ, Irwin CP, Fisher RS. Memory and mood outcomes after anterior thalamic stimulation for refractory partial epilepsy. Seizure. 2017;45:133-141.

7. Grewal SS, Middlebrooks EH, Kaufmann TJ, et al. Fast gray matter acquisition T1 inversion recovery MRI to delineate the mammillothalamic tract for preoperative direct targeting of the anterior nucleus of the thalamus for deep brain stimulation in epilepsy. Neurosurg Focus. 2018;45(2):E6.

8. Jiltsova E, Möttönen T, Fahlström M, et al. Imaging of anterior nucleus of thalamus using 1.5T MRI for deep brain stimulation targeting in refractory epilepsy. Neuromodulation. 2016;19(8):812-817.
9. Möttönen T, Katisko J, Haapasalo J, et al. The correlation between intraoperative microelectrode recording and 3-tesla MRI in patients undergoing ANT-DBS for refractory epilepsy. Stereotact Funct Neurosurg. 2016;94(2):86-92.

10. Koeppen JA, Nahravani F, Kramer M, et al. Electrical stimulation of the anterior thalamus for epilepsy: clinical outcome and analysis of efficient target. Neuromodulation. 2019;22(4): 465-471.

11. Lehtimäki K, Coenen VA, Gonçalves Ferreira A, et al. The surgical approach to the anterior nucleus of thalamus in patients with refractory epilepsy: experience from the international multicenter registry (MORE). Neurosurgery. 2019; 84(1):141-150.

12. Lehtimäki K, Möttönen T, Järventausta K, et al. Outcome based definition of the anterior thalamic deep brain stimulation target in refractory epilepsy. Brain Stimul. 2016;9(2):268-275.

13. Wang YC, Grewal SS, Middlebrooks EH, et al. Targeting analysis of a novel parietal approach for deep brain stimulation of the anterior nucleus of the thalamus for epilepsy. Epilepsy Res. 2019;153:1-6.

14. Rolston JD, Desai SA, Laxpati NG, Gross RE. Electrical stimulation for epilepsy: experimental approaches. Neurosurg Clin N Am. 2011;22(4):425-442, v.

15. McIntyre CC, Grill WM, Sherman DL, Thakor NV. Cellular effects of deep brain stimulation: model-based analysis of activation and inhibition. J Neurophysiol. 2004;91(4):1457-1469.

16. Laxpati NG, Kasoff WS, Gross RE. Deep brain stimulation for the treatment of epilepsy: circuits, targets, and trials. Neurotherapeutics. 2014;11(3):508-526.

17. Cheney PD, Griffin DM, Van Acker GM III. Neural hijacking: action of high-frequency electrical stimulation on cortical circuits. Neuroscientist. 2013;19(5):434-441.

18. Lee KH, Hitti FL, Chang SY, et al. High frequency stimulation abolishes thalamic network oscillations: an electrophysiological and computational analysis. J Neural Eng. 2011;8(4): 046001.

19. Chkhenkeli SA, Chkhenkeli IS. Effects of therapeutic stimulation of nucleus caudatus on epileptic electrical activity of brain in patients with intractable epilepsy. Stereotact Funct Neurosurg. 1997;69(1-4 Pt 2):221-224.

20. Dempsey EW, Morison RS. The production of rhythmically recurrent cortical potentials after localized thalamic stimulation. Am J Physiol. 1941;135(2):293-300.

21. Mirski MA, Rossell LA, Terry JB, Fisher RS. Anticonvulsant effect of anterior thalamic high frequency electrical stimulation in the rat. Epilepsy Res. 1997;28(2):89-100.

22. Kuncel AM, Grill WM. Selection of stimulus parameters for deep brain stimulation. Clin Neurophysiol. 2004;115(11): $2431-2441$.

23. Upton ARM, Amin I, Garnett S, et al. Evoked metabolic responses in the limbic-striate system produced by stimulation of anterior thalamic nucleus in man. Pacing Clin Electrophysiol. 1987;10(1 Pt 2):217-225.

24. Lim SN, Lee ST, Tsai YT, et al. Electrical stimulation of the anterior nucleus of the thalamus for intractable epilepsy: a long-term follow-up study. Epilepsia. 2007;48(2):342-347.

25. Lee KJ, Shon YM, Cho CB. Long-term outcome of anterior thalamic nucleus stimulation for intractable epilepsy. Stereotact Funct Neurosurg. 2012;90(6):379-385.

26. Oh YS, Kim HJ, Lee KJ, et al. Cognitive improvement after long-term electrical stimulation of bilateral anterior thalamic nucleus in refractory epilepsy patients. Seizure. 2012;21(3): $183-187$.

27. Kerrigan JF, Litt B, Fisher RS, et al. Electrical stimulation of the anterior nucleus of the thalamus for the treatment of intractable epilepsy. Epilepsia. 2004;45(4):346-354.

28. Lim SN, Lee ST, Tsai YT, et al. Long-term anterior thalamus stimulation for intractable epilepsy. Chang Gung Med J. 2008;31(3):287-296. 
29. Krishna V, King NKK, Sammartino F, et al. Anterior nucleus deep brain stimulation for refractory epilepsy: insights into patterns of seizure control and efficacious target. Neurosurgery. 2016;78(6):802-811.

30. Voges BR, Schmitt FC, Hamel W, et al. Deep brain stimulation of anterior nucleus thalami disrupts sleep in epilepsy patients. Epilepsia. 2015;56(8):e99-e103.

31. Kulju T, Haapasalo J, Lehtimäki K, et al. Similarities between the responses to ANT-DBS and prior VNS in refractory epilepsy. Brain Behav. 2018;8(6):e00983.

32. Van Gompel JJ, Klassen BT, Worrell GA, et al. Anterior nuclear deep brain stimulation guided by concordant hippocampal recording. Neurosurg Focus. 2015;38(6):E9.

33. Osorio I, Overman J, Giftakis J, Wilkinson SB. High frequency thalamic stimulation for inoperable mesial temporal epilepsy. Epilepsia. 2007;48(8):1561-1571.

34. Hodaie M, Wennberg RA, Dostrovsky JO, Lozano AM. Chronic anterior thalamus stimulation for intractable epilepsy. Epilepsia. 2002;43(6):603-608.

35. Horn A, Li N, Dembek TA, et al. Lead-DBS v2: towards a comprehensive pipeline for deep brain stimulation imaging. Neuroimage. 2019;184:293-316.

36. Avants BB, Epstein CL, Grossman M, Gee JC. Symmetric diffeomorphic image registration with cross-correlation: evaluating automated labeling of elderly and neurodegenerative brain. Med Image Anal. 2008;12(1):26-41.

37. Ewert S, Plettig P, Li N, et al. Toward defining deep brain stimulation targets in MNI space: a subcortical atlas based on multimodal MRI, histology and structural connectivity. Neuroimage. 2018;170:271-282.

38. Hartl E, Bötzel K, Mehrkens J-H, Noachtar S. Seizure reductions outlast DBS explantation. Brain Stimul. 2018;11(3): 636-638.

39. Li MCH, Cook MJ. Deep brain stimulation for drug-resistant epilepsy. Epilepsia. 2018;59(2):273-290.

40. Järvenpää S, Rosti-Otajärvi E, Rainesalo $S$, et al. Executive functions may predict outcome in deep brain stimulation of anterior nucleus of thalamus for treatment of refractory epilepsy. Front Neurol. 2018;9:324.

41. Hartikainen KM, Sun L, Polvivaara M, et al. Immediate effects of deep brain stimulation of anterior thalamic nuclei on executive functions and emotion-attention interaction in humans. J Clin Exp Neuropsychol. 2014;36(5):540-550.

42. Mirski MA, Fisher RS. Electrical stimulation of the mammillary nuclei increases seizure threshold to pentylenetetrazol in rats. Epilepsia. 1994;35(6):1309-1316.
43. Moruzzi G, Magoun HW. Brain stem reticular formation and activation of the EEG. Electroencephalogr Clin Neurophysiol. 1949;1(4):455-473.

44. Hamani C, Ewerton FIS, Bonilha SM, et al. Bilateral anterior thalamic nucleus lesions and high-frequency stimulation are protective against pilocarpine-induced seizures and status epilepticus. Neurosurgery. 2004;54(1):191-197.

45. Satzer D, Lanctin D, Eberly LE, Abosch A. Variation in deep brain stimulation electrode impedance over years following electrode implantation. Stereotact Funct Neurosurg. 2014; 92(2):94-102.

\section{Disclosures}

All authors are participating in clinical trials collaborated on by Medtronic (Medtronic Registry for Epilepsy [MORE], NCT01521754; Chronic Effects of DBS in Parkinson's Disease and Dystonia [Activa PC+S], NCT01934296). Dr. Mehrkens: honoraria from Medtronic, Abbott, Boston Scientific, and Brainlab. Drs. Kaufmann and Bötzel: speaker honoraria from Medtronic.

\section{Author Contributions}

Conception and design: Kaufmann, Noachtar. Acquisition of data: Kaufmann, Bötzel, Vollmar, Mehrkens. Analysis and interpretation of data: Kaufmann, Vollmar, Mehrkens. Drafting the article: Kaufmann. Critically revising the article: all authors. Reviewed submitted version of manuscript: Kaufmann, Vollmar, Mehrkens. Approved the final version of the manuscript on behalf of all authors: Kaufmann. Statistical analysis: Kaufmann. Administrative/technical/material support: Kaufmann, Bötzel, Mehrkens. Study supervision: Noachtar.

\section{Correspondence}

Elisabeth Kaufmann: Epilepsy Center, University Hospital, LMU Munich, Germany. elisabeth.kaufmann@med.uni-muenchen.de. 